\title{
Transformations properties of quantum optical signals in phase modulator
}

\author{
Alexander Trifanov* and Ekaterina Trifanova \\ ITMO University, Control Systems and Robotics, 197101 Saint Petersburg, Russia
}

\begin{abstract}
We investigate evolution of multimode quantum light in electro-optical modulator, using semiclassical model of phase modulation process. For the case of monochromatic incoming signal, we consider transformation of its spectrum and calculate statistical properties of each frequency mode. Particularly, we find that for the case of initial coherent state our result is covered by the well-known classical model. While for the case of initially squeezed state we observe single- and multi-mode squeezing in frequency spectrum of modulated light.
\end{abstract}

\section{Introduction}

Quantum communication systems and protocols based on the frequency bin codding are the most perspective in quantum information technologies due to the relatively simple hardware which basically consists of passive phase shifters and electro-optical phase modulators (EOPM) transforming frequency spectra of optical signals [1-2]. All of these requires theoretical models of phase modulation process generalized to the area of quantum states. There are some difficulties with unitarity which appears form straightforward generalization of existing classical model of electro-optical phase modulation process [3]. In [4] we suggest an algebraic model of EOPM based of effective Hamiltonian approach describing multimode quantum parametric process including arbitrary, but finite number of interacting modes. From that we obtain transformation of each frequency mode operator $a_{\mu}(t)$ following from temporal evolution of signal inside phase modulator [4]:

$$
a_{\mu}(t)=\sum_{v=-S}^{S}(-1)^{v} e^{-i(\mu+v) \alpha} e^{-i \gamma t} e^{-i(\mu-v) \varphi} d_{\mu \nu}^{(S)}(\beta) a_{v}=\sum_{v=-S}^{S} M_{\mu \nu}(\beta) a_{v} .
$$

Here $a_{v}$ is initial operator for $v$-th mode, $2 S+1$ is number of interacting modes, indexing form $-S$ to $S ; \alpha, \beta, \gamma, \varphi$ are some constants following form parameters of phase modulation process, and $d_{\mu \nu}^{(S)}(\beta)$ is a small Wigner d-matrix.

In this paper we deal with transformation properties of quantum state, which has incoming monochromatic signal with spectral index $v=0$ (odd number case) and frequency $\omega_{v}=\omega_{0}$, in EOPM taking into account cases of Fock, coherent, and squeezed states. For them we calculate spectrum transformation and statistical properties for each interacting spectrum mode and analyze "spreading" of initial quantum state among all frequency modes.

\footnotetext{
* Corresponding author: alextrifanov@gmail.com
} 


\section{State transformation}

In the following we will use inverse transformation, which with the property of incoming signal may be written through Legendre polynomials as follows:

$$
a_{0}=\sum_{\mu=-S}^{S} M_{0 \mu}^{*}(-\beta) a_{\mu}(t)=\sum_{\mu=-S}^{S} \sqrt{\frac{(S-\mu) !}{(S+\mu) !}} P_{\mu}^{(S)}(\cos \beta) e^{-i \mu \alpha} a_{\mu}(t),
$$

which show how initial mode evaluate in the superposition of bosonic modes operators.

\subsection{Coherent state}

Using Eq.(2) we can evaluate initial coherent state in time and obtain for all spectrum frequency modes (here $D_{0}(\alpha)$ is shift operator for mode with $v=0$ ):

$$
|\alpha\rangle_{0}=D_{0}(\alpha)|0\rangle \rightarrow \prod_{\mu=-S}^{S} D_{\mu}\left(M_{0 \mu}^{*}(-\beta) \alpha\right)|0\rangle \text {. }
$$

And we can conclude that during modulation process all modes "shift" to coherent state.

\subsection{Fock state}

For the case of Fock state we have to investigate the behaviour of $n$-th operator power under transformation from Eq.(2):

$$
|n\rangle=\frac{\left(a_{0}^{\dagger}\right)^{n}}{\sqrt{n !}}|0\rangle \rightarrow \frac{1}{\sqrt{n !}} \sum_{k_{1}+k_{2}+\ldots+k_{J}=n}\left(\begin{array}{c}
n \\
k_{1}, k_{2}, \ldots, k_{J}
\end{array}\right) \prod_{\mu=-S}^{S}\left[M_{\mu 0} a_{\mu}^{\dagger}\right]^{k_{\mu}}|0\rangle,
$$

which means that after modulation of monochromatic mode prepared in Fock state, resulting modulated state is in general entangled states of collective mode states with Fock conditional states of each mode.

\subsection{Squeezed state}

Finally, we consider squeezed state of incoming single mode signal:

$$
|\xi\rangle=S(\xi)|0\rangle \rightarrow \exp \left[\frac{1}{2}\left(A Z^{\dagger} A+A^{\dagger} Z A^{\dagger}\right)\right]|0\rangle,
$$

where $A=\left(a_{-S} \ldots a_{S}\right)^{T}$ and $Z$ is matrix of operator valued quadratic form. From here one can see that there are two process takes place in EOPM: single-mode and two-mode squeezing, which parameters are contained in matrix $Z \rightarrow Z^{*}=M_{0 \mu}^{*} M_{0 v}^{*}$. Applying polar decomposition $Z=R \cdot e^{i \Phi}$, we obtain parameters for each kind of squeezing.

This work was partially financially supported by the Government of the Russian Federation (grant 0808), grant 16-11-10330 of Russian Science Foundation.

\section{References}

1. Capmany J., Fernandez-Pousa C.R., IEEE Photonics Journal, 4 (6), 2074-2084 (2012)

2. Lukens J.M., Lougovsky P., Optica, 4 (1), 010008-09 (2017)

3. Capmany J., Fernandez-Pousa C.R., Laser Photonics Review, 5 (6), 750-772 (2011)

4. Miroshnichenko G.P. et.al., Journal of the Optical Society of America B, 34 (6), 061177-14 (2017) 